\title{
Identification of Chromosome 17 Trisomy in a Cynomolgus Monkey (Macaca fascicularis) by Multicolor FISH Techniques
}

\author{
Aoi Kimura $^{a}$ Satowa Yahashi ${ }^{a} \quad$ Fumio Chatani $^{a} \quad$ Hideyuki Tanabe $^{b}$ \\ ${ }^{a}$ Drug Safety Research Laboratories, Shin Nippon Biomedical Laboratories, Ltd., Kagoshima, Japan; bepartment of \\ Evolutionary Studies of Biosystems, School of Advanced Sciences, The Graduate University for Advanced Studies, \\ SOKENDAI, Hayama, Japan
}

\section{Keywords}

Cynomolgus monkey (Macaca fascicularis) · Trisomy 17 .

Patau syndrome $\cdot$ Multicolor FISH

\begin{abstract}
A female cynomolgus monkey (Macaca fascicularis) with facial features characteristic of Down syndrome showed abnormal behavior, unwariness toward humans, and poor concentration. The number of metaphase chromosomes in blood lymphocytes was examined and found to be 43 , which indicated one extra chromosome to the normal diploid number $(2 n=42)$. We then used Q-banding and multicolor FISH techniques to identify the extra chromosome. The results revealed an additional chromosome 17, with no other chromosomal rearrangements, such as translocations. Since no mosaicism or heterozygous variant chromosomes were observed, full trisomy 17 was assessed in this female cynomolgus monkey. Chromosome 17 corresponds to human chromosome 13, and human trisomy 13 , known as Patau syndrome, results in severe clinical signs and, often, a short life span; however, this individual has reached an age of 10 years with only mild clinical signs. Although genomic differences exist between human and macaques, this individual's case could help to reveal the pathological and genetic mechanisms of Patau syndrome.

(c) 2021 The Author(s)

Published by S. Karger AG, Basel
\end{abstract}

karger@karger.com www.karger.com/cgr

Karger"

GOPEN ACCESS
C 2021 The Author(s)

Published by S. Karger AG, Basel

This article is licensed under the Creative Commons Attribution 4.0 International License (CC BY) (http://www.karger.com/Services/ OpenAccessLicense). Usage, derivative works and distribution are permitted provided that proper credit is given to the author and the original publisher.

\section{Introduction}

Cynomolgus monkeys (Macaca fascicularis) are widely used for safety evaluation in drug development because they are physiologically and anatomically close to humans. Over 25,000 cynomolgus monkeys are bred and reared under the Shin Nippon Biomedical Laboratories, Ltd. Group. The facility in Kagoshima, Japan, has a constant stock of approximately 2,800 cynomolgus monkeys. One individual, a female, showed abnormal behavior, unwariness toward humans, and poor concentration during rearing. It was noted that this individual had quite similar facial features to those associated with Down syndrome, namely, increased distance between the eyes, upward slanting eyes, and flattened nose (Fig. 1). Therefore, we examined metaphase chromosomes in blood lymphocytes and found that the number of chromosomes was not 42 but 43 per cell, suggesting a trisomy. To identify the genetic composition of the extra chromosome, we performed Q-banding analysis and multicolor FISH with human chromosome-specific painting probes. Here, we report the results of our investigation on this rare case.
Correspondence to:

Aoi Kimura, kimura-aoi@snbl.co.jp

Hideyuki Tanabe, tanabe_hideyuki@ soken.ac.jp 


\section{Materials and Methods}

\section{Subject}

The female cynomolgus monkey (ID number K141516) was born on June 18, 2010, at the Cambodia Laboratory of Shin Nippon Biomedical Laboratories, Ltd. (SNBL). Its dam and sire were feral animals in origin. After arriving at the facility in Kagoshima at 4 years old, the animal showed abnormal behavior: low wariness toward humans, slow actions, inability to grasp feed between the thumb and forefinger, and poor concentration. Anemia often occurs in this animal due to excessive menstrual bleeding, but this recovers spontaneously. This individual is being reared according to the standard procedures at the Kagoshima site of SNBL, Drug Safety Research Laboratories.

\section{Cytogenetics}

Peripheral blood was obtained from the femoral vein using a heparinized tube. Metaphase spreads from blood lymphocytes were prepared according to the standard protocol [Tanabe et al., 1994], with slight modification. In brief, $7.5 \%$ total blood was cultured in RPMI 1640 medium supplemented with 10\% heat-inactivated fetal bovine serum and $2.5 \%$ phytohemagglutinin, $\mathrm{M}$ form (Gibco 10576015) for $70 \mathrm{~h}$ at $37^{\circ} \mathrm{C}$. Colcemid solution (Kary$\mathrm{oMAX}^{\mathrm{TM}}$, Gibco 15210040) was applied at a final concentration of $0.04 \mu \mathrm{g} / \mathrm{mL}$ for the final $2 \mathrm{~h}$ of incubation. Harvested cells were suspended in a hypotonic $0.075 \mathrm{M} \mathrm{KCI}$ solution with sodium citrate $(0.05 \% ; \mathrm{w} / \mathrm{v})$ for $25 \mathrm{~min}$ at $37^{\circ} \mathrm{C}$ and fixed with methanol:acetic acid (3:1) fixative. Metaphase spreads were obtained by using HANABI metaphase spreader (ADSTEC Corporation). The slides were stained with $2.5 \%$ Giemsa solution (Merck 109204) or 2\% DAPI in $2 \times$ SSC, and then photographs were taken for counting the number of metaphase chromosomes. As a control, a normal female cynomolgus monkey of similar weight and age to the abnormal monkey was selected, and the number of chromosomes was determined. To identify the trisomic chromosome, we performed QFQ- or QFH-banding [Caspersson et al., 1970; Yoshida et al., 1975]. We assigned the macaque chromosome numbers according to the nomenclature [Rogers et al., 2006], which was slightly modified from the originally proposed idiogram of banded chromosomes in macaque species [Small et al., 1985; Wienberg et al., 1992].

\section{Fluorescence in situ Hybridization}

FISH was conducted as described in Tanabe et al. [1996] and Omori et al. [2017], with some modifications. Human chromosome-specific and chromosome arm-specific painting probes were kindly provided by Prof. Dr. Thomas Cremer (LMU, Munich, Germany). We used 13q, 16, 17, 18, 19, 21q-specific DNA probes, and probe labeling was performed by DOP-PCR [Telenius et al., 1992] in the presence of biotin-16-dUTP (Roche 11093711103), digoxigenin-11-dUTP (Roche 11093088910), or dinitrophenol-11-dUTP (PerkinElmer NEL551). Combinatorially labeled probes were mixed with human Cot-1 DNA, subjected to ethanol precipitation, and resuspended in hybridization solution (50\% formamide and $10 \%$ dextran sulfate in $2 \times$ SSC). After hybridization for approximately $24-36 \mathrm{~h}$, the slides were washed twice in $2 \times$ SSC, 3 times for $5 \mathrm{~min}$ in $0.1 \times \mathrm{SSC}$ at $62.5^{\circ} \mathrm{C}$, and for $2 \mathrm{~min}$ in $4 \times \mathrm{SSC}$. The metaphase cells were then blocked in $4 \times$ SSC containing 5\% BSA and $0.2 \%$ Tween- 20 for $20 \mathrm{~min}$ at $37^{\circ} \mathrm{C}$ prior to probe detection. Probe detection was performed according to the standard procedure us- ing avidin-Cy5 (Jackson ImmunoResearch), rabbit anti-DNP antibody (Sigma), and mouse anti-digoxigenin antibody (Sigma) in the first layer, and goat anti-rabbit Alexa 488-conjugated antibody (Molecular Probes) and sheep anti-mouse Cy3-conjugated antibody (Jackson ImmunoResearch) in the second layer. The slides were counterstained with DAPI, and mounted in Vectashield Antifade (Vector). For multiplex FISH analysis [Speicher et al., 1996], 24XCyte human mFISH probes (MetaSystems), chromosomespecific painting probes combinatorially labeled with 5 fluorochromes, were used according to the manufacturer's protocol. FISH images were captured and analyzed using a Leica DM5000B fluorescent microscope equipped with a CCD camera and CW4000 image analysis software (Leica Microsystems).

\section{Results}

Counting the number of chromosomes derived from female monkey K141516 showed that the incidence of 43 chromosomes was high, with a frequency of $95.9 \%$ (116/121). Among the 121 metaphases observed in total, 116 metaphases had 43 chromosomes, and the number of chromosomes in the remaining 5 metaphases ranged from 37 to 42 . One of the causes for these lower numbers was considered to be the loss of chromosomes during preparation of the metaphase spreads. The diploid chromosome number of macaque species has been reported as $2 n=42$ [Fernandez-Donoso et al., 1970]; therefore, the obtained counting results suggested that female monkey K141516 has full trisomy, possessing one extra chromosome without mosaicism. To identify the trisomic chromosome, QFQ- or QFH-banding was performed, and the extra chromosome was found to be a small submetacentric chromosomes. The candidate chromosomes were 16 , $17,18,19$, and 20 , which are homologous to human chromosomes 17, 13, 18, 19, and 16, respectively. Therefore, we performed multicolor FISH with human chromosome-specific painting probes to precisely identify the trisomic chromosome. Using combinatorially labeled painting probes specific for human chromosomes 13q, 16, 17, 18 , and 19 , three signals were only detected with the chromosome 13q probe. No split signals were observed with the painting probes; specifically, no chromosomal rearrangements, such as translocations, were observed. In addition, we used human chromosome 13, 18, and 21 probes in multicolor FISH because these 3 chromosomes are common human trisomic chromosomes and because we had noted facial features similar to those in humans with Down syndrome, trisomy 21 (Fig. 1). The results showed that 2 chromosomes each were painted by the chromosome 18 and $21 \mathrm{q}$ probes, while the chromosome $13 \mathrm{q}$ probe labeled 3 chromosomes (Fig. 2). In addition, we 

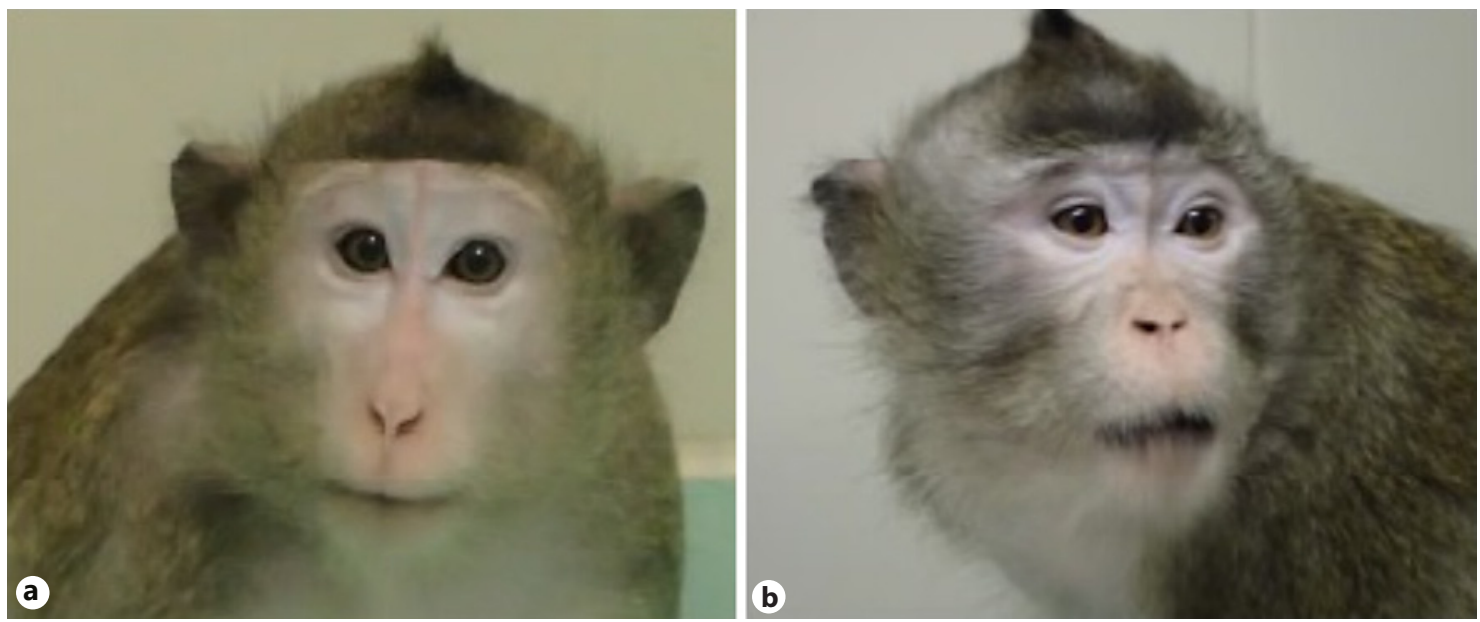

Fig. 1. Facial features of cynomolgus monkeys. a Normal female monkey at the age of 6 years. b Female trisomy monkey K141516 with abnormal appearance at 6 years old.

Fig. 2. Multicolor FISH on a metaphase plate of cynomolgus monkey K141516 using painting probes for human chromosomes $13 \mathrm{q}, 18$, and 21q. a Metaphase counterstained with DAPI. b Three-color FISH image. Two green signals represent a pair of homologous regions of cynomolgus monkey chromosome 3 corresponding to human chromosome 21. Two light blue signals indicate cynomolgus monkey chromosome pair 18 corresponding to human chromosome 18. Three magenta signals label trisomic cynomolgus monkey chromosomes 17 , corresponding to human chromosome 13. Asterisks indicate the trisomic chromosomes.
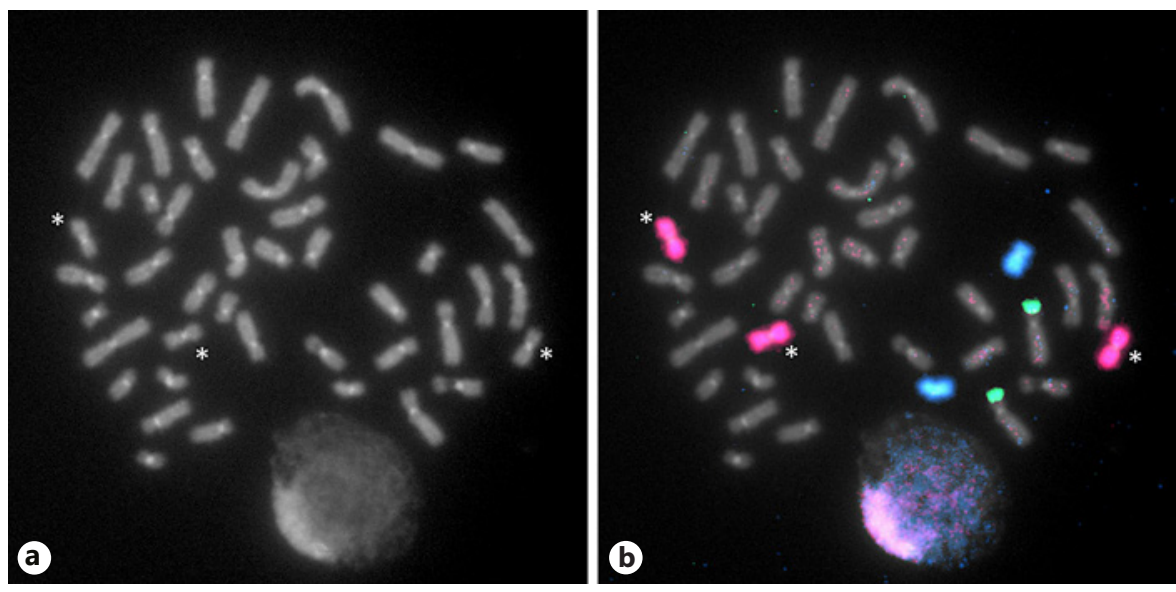

conducted multiplex FISH using 24XCyte probes and confirmed that only the chromosome 13 probe marked 3 chromosomes (Fig. 3). All other probes painted only 2 chromosomes, indicating disomy. These results suggested that monkey K141516 has full trisomy of chromosome 17, which is homologous to human chromosome 13.

\section{Discussion and Conclusion}

Human trisomy 13 is the third most common autosomal numerical abnormality at birth, after trisomy 21 and trisomy 18. Human trisomy 13 is known as Patau syndrome [Patau et al., 1960]. It occurs at an incidence of approximately 1:5,000-7,000 live births in humans [Magenis et al., 1968; Baty et al., 1994]. Common signs of trisomy 13 may include holoprosencephaly (failure of the forebrain to di- vide properly), midline anomalies, structural eye defects (microphthalmia and retinal dysplasia), heart defects, and severe intellectual disability. Facial anomalies such as cleft lip, cleft palate, and auricular morphological aberration are also common. Approximately $80 \%$ of patients have severe congenital cardiovascular anomalies and high juvenile mortality (death by 1 year of age). Monkey K141516 with trisomy 17 in the present study did not show morphological facial or cardiovascular anomalies typical of Patau syndrome in humans, but did show minor structural abnormalities in the eyes. Follow-up research revealed a partially cloudy macula and larger than normal optic disc cupping (coloboma). In functional status, poor use of the thumb, slow actions, and a deficit in learning ability were also observed. Although high juvenile mortality has been reported in humans with Patau syndrome, monkey K141516 has just reached an age of 10 years without severe clinical signs. This 
Fig. 3. Multiplex FISH (M-FISH) on a metaphase of cynomolgus monkey K141516. a Metaphase counterstained with DAPI. b M-FISH image after hybridization with 24XCyte (MetaSystems) painting probes. Three painting signals represent the trisomic chromosomes 17 (asterisks), which were detected by the human chromosome 13 probe.
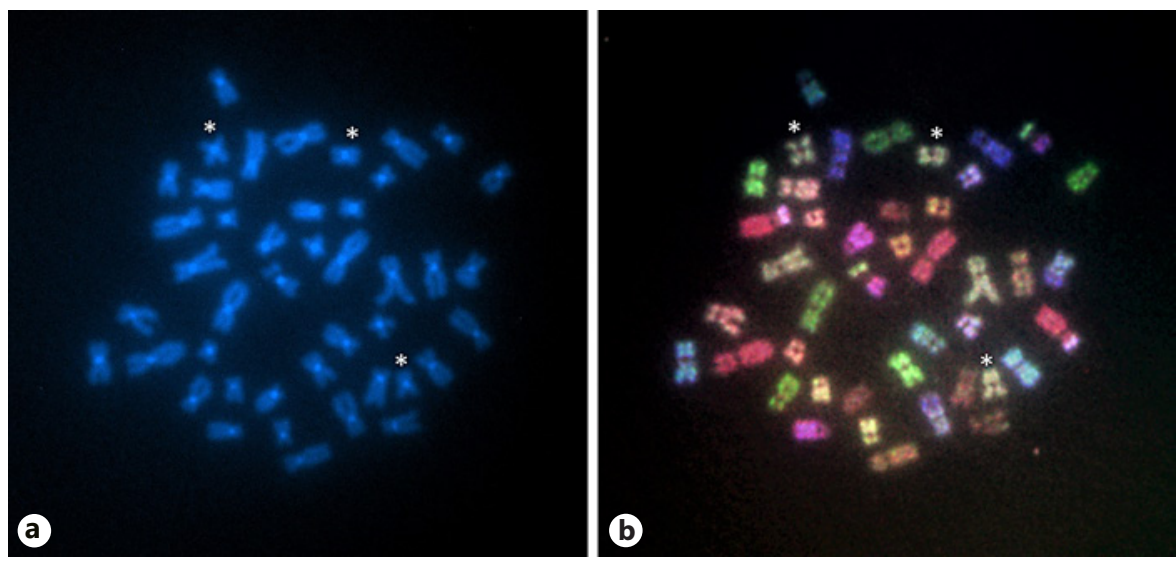

Table 1. Summary of reported cases with trisomies in primates

\begin{tabular}{|c|c|c|c|}
\hline $\begin{array}{l}\text { Human } \\
\text { trisomies }\end{array}$ & $\begin{array}{l}\text { Primate } \\
\text { trisomies }\end{array}$ & Physical and/or behavioral features & References \\
\hline \multirow[t]{2}{*}{ HSA21 } & PTR22 & Clinically and behaviorally like Down syndrome & McClure et al., 1969 \\
\hline & PPY22 & Clinically and behaviorally abnormal & Andrle et al., 1979 \\
\hline \multirow[t]{3}{*}{ HSA18 } & PPA17 & Multiple congenital abnormalities & Lear et al., 2001 \\
\hline & MNE18 & Inappropriate social and motor behavior & Swartz and Sackett, 1994 \\
\hline & MMU18 & Hydrocephalic affliction, motor deficiencies, delay of social behavior & de Waal et al., 1996 \\
\hline \multirow[t]{3}{*}{ HSA13 } & PHA17 & Polydactyly of the forelimbs, patent foramen ovale, pylectasis & Moore et al., 2007 \\
\hline & MFA16 & Slow action, flatter face, repetitive behavior & Ward et al., 1994 \\
\hline & MNE16 & $\begin{array}{l}\text { Mosaic karyotype, } 75.7 \% \text { trisomy, } 11.2 \% \text { disomy, developmental delay, mental retardation, cleft } \\
\text { lip, situs inversus of the heart }\end{array}$ & Vigfusson et al., 1986 \\
\hline
\end{tabular}

HSA, Homo sapiens; PTR, Pan troglodytes; PPA, Pan paniscus; PPY, Pongo pygmaeus; PHA, Papio hamadryas; AAB, Aotus azarae boliviensis; ALG, Aotus lemurinus griseimembra; MMU, Macaca mulatta; MNE, Macaca nemestrina; MFA, Macaca fascicularis.

long life is probably not only due to an absence of severe abnormalities in morphology and systematic function, but also the differences in genomic content between human chromosome 13 and cynomolgus monkey chromosome 17.

In non-human primates, several cases of autosomal trisomies corresponding to human trisomies 21,18 , and 13 have been reported, such as chimpanzee trisomy 22 [McClure et al., 1969; Hirata et al., 2017], bonobo trisomy 17 [Lear et al., 2001], orangutan trisomy 22 [Andrle et al., 1979], baboon trisomies 17 and 18 [Howell et al., 2006; Moore et al., 2007], and night monkey trisomy 18 [Hirai et al., 2017]. In macaques, 2 cases homologous to human trisomy 18 [Swartz and Sackett, 1994; de Waal et al., 1996] and 3 cases homologous to human trisomy 13 [Vigfusson et al., 1986; Ward et al., 1994; Ruppenthal et al., 2004] have been reported. Cases of trisomy 17 in Macaca nemestrina as well as trisomy 18 in Macaca mulatta are likely to result in severe signs and multiple anomalies during development, even in mosaic individuals; however, cases of trisomy 17 in $M$. fascicularis do not show the severe trisomy 13 features seen in humans. This characteristic phenotype can be associated with the genomic structure of cynomolgus monkey chromosome 17, which is unique among macaque species (https://genome.ucsc.edu/cgi-bin/hgGateway). These cases are summarized in Table 1. According to extensive comparative BAC-FISH analysis of all chromosomes between macaques and humans, the present centromere on macaque chromosome 17 , which corre- 
sponds to the human chromosome $13 \mathrm{q} 21.31$ region, is derived from the neocentromere that formed during the course of primate evolution [Cardone et al., 2006; Ventura et al., 2007; Stanyon et al., 2008; Sangpakdee et al., 2018]. This suggests that the present human chromosome 13 conserved the syntenic organization of the primate ancestral chromosome and that deletion of rDNA loci, centromere inactivation, and neocentromere formation (centromere repositioning) with the duplication of approximately $427 \mathrm{~kb}$ corresponding to human 13q21.31 $(61,282,357-61,709,544)$ surrounding the neocentromere have successively occurred in the Old World monkey branches [Cardone et al., 2006; Sangpakdee et al., 2018]. During these events, loss of chromatin involving the NOR region and/or the appearance of neocentromeres with the additional $427 \mathrm{~kb}$ could have affected the total status of gene expression in the macaque chromosome 17. Within the duplicated $427-\mathrm{kb}$ segment there are no coding genes, but several long noncoding RNAs are present. Therefore, a possible implication would be that these ncRNAs contribute to decrease the severe clinical signs, especially in M. fascicularis; however, it is still unknown why severe clinical signs are retained in M. nemestrina (Table 1). Probably $3 \mathrm{D}$ organization of the chromatin with regard to dynamic alteration of topologically associating domains and compartments near the neocentromeric DNA region at $13 \mathrm{q} 21.31$ could affect the status of gene expression on the macaque chromosome 17 [Cardone et al., 2006; Lazar et al., 2018]. Thus, detailed analyses of the genetic differences between $M$. fascicularis and M. nemestrina would be important for understanding the mechanisms attenuating the clinical signs and the genetic basis of cynomolgus monkey trisomy 17 without Patau syndrome lethality.

\section{Acknowledgement}

The authors are grateful to Prof. Dr. Thomas Cremer, LMU, Munich, for kindly providing human chromosome arm-specific painting DNA probes.

\section{Statement of Ethics}

This animal research was conducted according to ethical standards in Shin Nippon Biomedical Laboratories, Ltd., Drug Safety Research Laboratories, which is accredited by AAALAC International.

\section{Conflict of Interest Statement}

The authors have no conflicts of interest to declare.

\section{Funding Sources}

This study was supported in part by grants from the International Collaborative Research Project, "Integrated Anthropology," for School of Advanced Sciences, The Graduate University for Advanced Studies, SOKENDAI.

\section{Author Contributions}

A.K. made contributions to performing cytogenetic and FISH analyses, and drafting the manuscript. S.Y. made contributions to finding the K141516 individual and promoting the conception and design of the study. F.C. participated in drafting the manuscript and revising it critically for important intellectual content. H.T. made contributions to performing cytogenetics and FISH analyses, and drafting the manuscript for the final version, ensuring that the experimental works are appropriately investigated.

\section{References}

Andrle M, Fiedler W, Rett A, Ambros P, Schweizer D. A case of trisomy 22 in Pongo pygmaeus. Cytogenet Cell Genet. 1979;24(1):1-6.

Baty BJ, Blackburm BL, Carey JC. Natural history of trisomy 18 and trisomy 13. I. Growth, physical assessment, medical histories, survival, and recurrence risk. Am J Med Genet. 1994; 49(2):175-88.

Cardone MF, Alonso A, Pazienza M, Ventura M, Montemurro G, Carbone L, et al. Independent centromere formation in a capricious, gene-free domain of chromosome 13q21 in Old World monkeys and pigs. Genome Biol. 2006;7(10):R91.

Caspersson T, Zech L, Johansson C, Modest EJ. Identification of human chromosomes by DNA-binding fluorescent agents. Chromosoma. 1970;30(2):215-27. de Waal FB, Uno H, Luttrell LM, Meisner LF, Jeannotte LA. Behavioral retardation in a macaque with autosomal trisomy and aging mother. Am J Ment Retard. 1996;100(4):37890.

Fernandez-Donoso R, Lindsten J, Norrby E. The chromosomes of the cynomolgus macaque (Macaca fascicularis). Hereditas. 1970;65(2): 269-75.

Hirai H, Hirai Y, Morimoto M, Kaneko A, Kamanaka Y, Koga A. Night monkey hybrids exhibit de novo genomic and karyotypic alterations: The first such case in primates. Genome Biol Evol. 2017;9(4):945-55.

Hirata S, Hirai H, Nogami E, Morimura N, Udono T. Chimpanzee Down syndrome: a case study of trisomy 22 in a captive chimpanzee. Primates. 2017;58(2):267-73.
Howell KH, Hubbard GB, Moore CM, Dunn BG, von Kap-Herr C, Raveendran M, et al. Trisomy of chromosome 18 in the baboon (Papio hamadryas anubis). Cytogenet Genome Res. 2006;112(1-2):76-81.

Lazar NH, Nevonen KA, O'Connell B, McCann C, O'Neill RJ, Green RE, et al. Epigenetic maintenance of topological domains in the highly rearranged gibbon genome. Genome Res. 2018;28(7):983-97.

Lear TL, Houck ML, Zhang YW, Debnar LA, Sutherland-Smith MR, Young L, et al. Trisomy 17 in a bonobo (Pan paniscus) and deletion of $3 \mathrm{q}$ in a lowland gorilla (Gorilla gorilla gorilla): Comparison with human trisomy 18 and human deletion 4q syndrome. Cytogenet Cell Genet. 2001;95(3-4):22833. 
Magenis RE, Hecht F, Milham S Jr. Trisomy 13 (D1) syndrome: studies on parental age, sex ratio, and survival. J Pediatr. 1968;73(2):2228.

McClure HM, Belden KH, Pieper WA, Jacobson CB. Autosomal trisomy in a chimpanzee: resemblance to Down's syndrome. Science. 1969;165(3897):1010-2.

Moore CM, Hubbard GB, Dick E, Dunn BG, Raveendran M, Rogers J, et al. Trisomy 17 in a baboon (Papio hamadryas) with polydactyly, patent foramen ovale and pyelectasis. Am J Primatol. 2007;69(10):1105-18.

Omori S, Tanabe H, Banno K, Tsuji A, Nawa N, Hirata K, et al. A pair of maternal chromosomes derived from meiotic nondisjunction in trisomy 21 affects nuclear architecture and transcriptional regulation. Sci Rep. 2017;7(1): 764.

Patau K, Smith DW, Therman E, Inhorn SL, Wagner HP. Multiple congenital anomaly caused by an extra autosome. Lancet. 1960;1(7128): 790-3.

Rogers J, Garcia R, Shelledy W, Kaplan J, Arya A, Johnson Z, et al. An initial genetic linkage map of the rhesus macaque (Macaca mulatta) genome using human microsatellite loci. Genomics. 2006;87(1):30-8.

Ruppenthal GC, Moore CM, Best RG, WalkerGelatt CG, Delio PJ, Sackett GP. Trisomy 16 in a pigtailed macaque (M. nemestrina) with multiple anomalies and developmental delays. Am J Ment Retard. 2004;109(1):9-20.
Sangpakdee W, Tanomtong A, Chaveerach A, Pinthong K, Trifonov V, Loth K, et al. Molecular cytogenetic analysis of one African and five Asian macaque species reveals identical karyotypes as in mandrill. Curr Genomics. 2018;19(3):207-15.

Small M, Stanyon R, Smith DG, Sineo L. Highresolution chromosomes of rhesus macaques (Macaca mulatta). Am J Primatol. 1985;9(1): 63-7.

Speicher MR, Ballard GS, Ward DC. Karyotyping human chromosomes by combinatorial multi-fluor FISH. Nat Genet. 1996;12(4): 368-75.

Stanyon R, Rocchi M, Capozzi O, Roberto R, Misceo D, Ventura M, et al. Primate chromosome evolution: ancestral karyotypes, marker order and neocentromeres. Chromosome Res. 2008;16(1):17-39.

Swartz KB, Sackett GP. Social preferences by and for pigtailed macaques (Macaca nemestrina) with trisomy 18. Am J Ment Retard. 1994; 99(2):141-50.

Tanabe H, Ishida T, Ueda S, Sofuni T, Mizusawa $\mathrm{H}$. Regional assignment of the human immunoglobulin processed pseudogene $\mathrm{C}$ epsilon 3 (IGHEP2) to 9p24.2-->p24.1 by fluorescence in situ hybridization. Cytogenet Cell Genet. 1994;66(2):93-5.

Tanabe H, Ishida T, Ueda S, Sofuni T, Mizusawa H. Molecular anatomy of human chromosome 9: Comparative mapping of the immunoglobulin processed pseudogene $\mathrm{C}$ epsilon 3 (IGHEP2) in primates. Cytogenet Cell Genet. 1996;73(1-2):92-6.
Telenius H, Carter NP, Bebb CE, Nordenskjold M, Ponder BA, Tunnacliffe A. Degenerate oligonucleotide-primed PCR: general amplification of target DNA by a single degenerate primer. Genomics. 1992;13(3):718-25.

Ventura M, Antonacci F, Cardone MF, Stanyon R, D'Addabbo P, Cellamare A, et al. Evolutionary formation of new centromeres in macaque. Science. 2007;316(5822):243-6.

Vigfusson NV, Dawson RJ, Jones JF, Shaw KJ, Lloyd MA, Ruppenthal GC, et al. Mosaic variegated trisomy $(42, \mathrm{XY} / 43, \mathrm{XY}$, + variable $)$ in a male pigtail macaque monkey. Cytogenet Cell Genet. 1986;42(3):154-8.

Ward OG, Miller RL, Johnson EH, Lucas JN, Meyne J. Identification of trisomy in Macaca fascicularis by fluorescence in situ hybridization with a human chromosome 13 DNA library. Hum Genet. 1994;94(3):247-51.

Wienberg J, Stanyon R, Jauch A, Cremer T. Homologies in human and Macaca fuscata chromosomes revealed by in situ suppression hybridization with human chromosome specific DNA libraries. Chromosoma. 1992;101(5-6): 265-70.

Yoshida MC, Ikeuchi T, Sasaki M. Differential staining of parental chromosomes in interspecific cell hybrids with a combined quinacrine and 33258 Hoechst technique. Proc Jpn Acad. 1975;51(3):184-7. 